\title{
Influence of Tree Cropping Systems on Physical Properties of Salt Affected Soil
}

\author{
C.S. Pandey* \\ Department of Horticulture, Narendra Deva University of Agriculture and Technology, \\ Kumarganj, Faizabad (U.P.), India \\ *Corresponding author
}

\section{A B S T R A C T}

\section{Keywords \\ Fruit trees, Aonla, Ber, Guava, Phalsa, Subabool, EC, pH, ESP and CEC, Canopy \\ Article Info \\ Accepted: \\ 07 March 2019 \\ Available Online: \\ 10 April 2019}

\begin{abstract}
The present investigation entitled "Influence of tree cropping systems on physical properties of salt affected soil" was carried out at wasteland management farm Akma, Department of Horticulture, Narendra Deva University of Agriculture and Technology, Narendra Nagar, Kumarganj, Faizabad, U.P. during the year 2010-11. All the tree cropping systems viz. Aonla + Ber, Aonla + Guava, Ber + Guava + Phalsa, Aonla + Karonda and Aonla + Subabool improved the physical properties of soil as compared to control (barren site). Decrease in bulk density, particle density, silt and increase in water holding capacity, hydraulic conductivity and sand percent indicated considerable improvement in physical properties of soil. Improvement was noticed more in inside canopy as compared to open side canopy of plants. The impact was recorded higher in upper depth as compared to lower depth of soil.
\end{abstract}

\section{Introduction}

Land is one of the limited and inelastic resources, which is reached to the state of high degree of degradation and shrinkage due to over exploitation and subsequent incensement of population growth and consequential anthropogenic pressure. Out of the total geographical area of 329 million ha, nearly 175 million ha area is suffering from different levels of degradation. Among degraded wastelands (173.08 million ha.), 7.0 million ha is adversely affected with excessive soluble salts/exchangeable sodium salts, which is unsuitable to profitable crop production. In Uttar Pradesh, out of 135.25 lakh ha degraded lands (46\% of total geographical area), 12.0 lakh ha area is under sodic lands, which is difficult to manage for cultivation of agronomical crops with optimal crop harvest (Agrawal and Gupta, 1968). The majority of such lands is lying barren and has become ecologically and economically unproductive due to chronic degradation and denudation. The presence of excessive salt in soil decreases its productivity as the salt inhibits not only the plant growth, but also deteriorates physical and chemical properties of soil. The absorption of sodium salt in such soil results into compact soil structure, low availability of essential plant nutrients and reduced microbial activities in soils, which 
inhibits the growth activities of most of the corps/plants. Vegetation exerts a decisive influence on physical and chemical characteristics and content of organic matter through their deep root system and addition of organic matter from biomass (Nandagouthar et al.,). The utilization and management of such categories of waste lands needs a systematic approach of land and crop management for effective and selective crop planning. The research work carried out earlier has shown that the reclamation of salt affected soils for cultivation of agronomical crop is not only expensive but management of such soil further becomes more complicated. A number of fruit trees species have been identified to have tolerance to sodic soil. The degree of tolerance to different levels of sodicity have been worked out in aonla, ber, bael, citrus, grape, guava, karonda, phalsa, and other horticultural crops by several scientist at different places. The effect of combined plantations of the selective fruit tree species at specific age (12 year old plantation) for improvement of salt affected and poor fertile soils has not been worked out so far. Therefore, there is great importance to study in systematic manner for improvement of chemical properties of salt affected soils through tree plantation as efficient and effective methods of land reclamation. The present investigation has been undertaken with objectives to study the changes in fertility status of soil and to find out the most suitable and potential cropping model for reclamation of salt affected soil.

\section{Materials and Methods}

The present investigation was undertaken at the Wasteland Management Farm, Akma, Narendra Deva University of Agriculture and Technology, Kumarganj, Faizabad (U.P.) during the year 2010-11. The 12 year old five selective tolerant fruit species viz. Aonla, Ber, Guava, Phalsa, Karonda and one fodder species subabool planted in different cropping models in an area of 0.5 ha each under wasteland conditions having alkali/sodic soils and poor soil fertility status were taken under study. The plant spacing was kept 8 meter for Aonla, Guava, Ber and Phalsa and 4 meter for Karonda and Subabool. The pit size was $1 \mathrm{~m}^{3}$ and filling mixtures were applied as per recommendations for sodic land plantation. The cultural practices were followed as per recommendations. The details of treatment are given below:

\section{Treatments}

The tree cropping systems -5

$$
\begin{aligned}
& \text { Aonla }+\operatorname{Ber}\left(\mathrm{C}_{1}\right) \\
& \text { Aonla }+ \text { Guava }\left(\mathrm{C}_{2}\right) \\
& \text { Aonla }+ \text { Karonda }\left(\mathrm{C}_{3}\right) \\
& \text { Ber }+ \text { Guava }+ \text { Phalsa }\left(\mathrm{C}_{4}\right) \\
& \text { Aonla }+ \text { Subabool }\left(\mathrm{C}_{5}\right)
\end{aligned}
$$

Sampling sites -2

Inside tree canopy (Basin)

Outside tree canopy (Centre)

Control - 1 (bare site)

Total treatment $=5 \times 2+1=11$

Design - Randomized Block Design (RBD)

Replication -4

Plot size -0.5 ha each.

\section{Collection of soil samples}

The soil samples were collected from each plot of cropping model and control plot (barren site). The soil samples were taken randomly from the basin (inside canopy) of each tree species and between the centre of rows and plants from 4 selected spots of each 
model at the depth of $0-15,15-30,30-45$ and 45-60 with the help of spade, khurpi, and soil serew auger after offset of mansoon i.e. in the month of October - November. The soil samples were collected from the inner rows of tree species by covering 4 trees $(2+2)$ of Aonla + Ber, Aonla + Guava, 8 trees $(2+2+$ 4) of Ber + Guava + Phalsa, 8 trees $(2+6)$ of Aonla + Karonda and Aonla + Subabool.

\section{Preparation of soil samples}

The samples collected from the plots of different models were brought to the laboratory and fresh weight was taken. The samples were dried in the shade at room temperature as well as in oven at $105^{\circ} \mathrm{C}$ temperature for 24 hours till the constant weight. Thereafter, each sample was grinded and sieved with 20 meshes and used for analysis of physical properties of soil as influenced by different cropping models.

\section{Observations recorded}

Chemical characteristics of soil samples were determined to find out the changes in chemical properties of soil as affected by each cropping models. The methods employed for estimation of changes in Physical properties of soil is mentioned below:

\section{Effect of tree cropping systems on physical properties of salt affected soil}

\section{Texture}

Present studies indicated that the texture of soil was considerably improved with all the tree cropping systems as compared to control. Mechanical analysis of soil under investigation revealed that it contains relatively higher proportion of silt and clay fraction and low proportion of sand making soil more compact and calcareous due to presence of calcium carbonate which makes it unfavourable for growth and development of most of the crop/plants. It is well known fact that texture of soil has marked influences on physical condition of soil which ultimately affects the cultural operations, water and nutrient availability to plants.

Our findings showed tremendous improvement in sand percentage and marked reduction in silt and clay percentage at all the depths in various cropping systems over control (barren site) making soil more porous and permeable. However, the differences were smaller between basins and centres of different models at respective depths. It may be possibly due to similar parent material of the experimental field. The reduced proportion of silt and clay with simultaneously increased proportion of sand might be due to increased accumulation of organic matter with plant age as a result of decomposition of litter fall, dead roots and other biomass. It could aid the formation of larger fraction of sand from the silt and clay factions. Similar results were also reported by Kamara and Haque (1992).

\section{Water holding capacity}

Observations pertaining to water holding capacity of soil showed that it improved significantly under different cropping systems at all the depths over control (barren site), but decreased with increasing depths of soil. Possible reason for it appears to be due to increased accumulation of organic matter resulting from decomposition of biomass (litter fall, dead roots etc.) and thereby, improvement in porosity, permeability and chemical properties of soil.

The maximum water holding capacity was found in Ber + Guava + Phalsa followed by Aonla + Ber cropping system which seems the matter of input from trees combination. Similar results were also reported by Khan 
(1966), Garg and Jain (1992), Khan (199798), Malviya and Singh (1998) and Singh et al., (2001) who found that water holding capacity of soil increased with plants association as compared to bare site.

\section{Bulk density}

Findings indicated that bulk density of soil was found to be reduced significantly with all the cropping systems at all the depths as compared with control (barren site). Studies also indicated that influence of different cropping systems was reduced with increase in depths of soil. It was minimum in Ber + Guava + Phalsa followed by Aonla + Ber cropping systems. Basins of plants under different combinations had lower bulk density as compared to centres. Increase in organic matter as a result of decomposition of leaf litter and other biomass with plant age and canopy may be main cause of reduced bulk density.

Mishra et al., (1988) also reported that bulk density decreased with tree association which resulted marked improvement in physical properties of soil. The increased porosity in the present investigation may also be one of the reasons for decreased bulk density of soil. The findings of Khan (1997-98), Malviya and Singh (1998) and Singh et al., (2001) also supported this fact that bulk density is considerably reduced under the plantation as compared to bare sites.

Table.1 Details of the cropping systems and plant population

\begin{tabular}{|c|c|c|c|c|}
\hline S. No. & Tree cropping systems & Plot size (ha) & $\begin{array}{c}\text { Total No of Plants } \\
\text { per plot }\end{array}$ & $\begin{array}{c}\text { Plant density } \\
\text { /ha. }\end{array}$ \\
\hline 1 & Aonla $+\operatorname{Ber}\left(\mathrm{C}_{1}\right)$ & 0.5 & $\begin{array}{l}\text { Aonla }=72 \\
\text { Ber }=50\end{array}$ & $\begin{array}{l}144 \\
100\end{array}$ \\
\hline 2 & Aonla + Guava $\left(\mathrm{C}_{2}\right)$ & 0.5 & $\begin{array}{l}\text { Aonla }=72 \\
\text { Guava }=50\end{array}$ & $\begin{array}{l}144 \\
100\end{array}$ \\
\hline 3 & Aonla + Karonda $\left(\mathrm{C}_{3}\right)$ & 0.5 & $\begin{array}{l}\text { Aonla }=72 \\
\text { Karonda }=110\end{array}$ & $\begin{array}{l}144 \\
220\end{array}$ \\
\hline 4 & Ber + Guava + Phalsa $\left(\mathrm{C}_{4}\right)$ & 0.5 & $\begin{array}{l}\text { Ber }=50 \\
\text { Guava }=72 \\
\text { Phalsa }=60\end{array}$ & $\begin{array}{l}100 \\
144 \\
120\end{array}$ \\
\hline 5 & Aonla + Subabool $\left(\mathrm{C}_{5}\right)$ & 0.5 & $\begin{array}{l}\text { Aonla }=72 \\
\text { Subabool }=110\end{array}$ & $\begin{array}{l}144 \\
220\end{array}$ \\
\hline 6 & Control $\left(\mathrm{C}_{0}\right)$ & 0.5 & Nil & Nil \\
\hline
\end{tabular}

Table.2 Methods used in analysis of physical properties of soil

\begin{tabular}{|l|l|}
\hline Soil properties & Methods adopted \\
\hline (a) Physical properties & \\
\hline (i) $\quad$ Soil texture & Bouyoucos hydrometer methods (Black, 1965) \\
\hline (ii) Water holding capacity & Method adopted by Chopra and Kanwar (1976) \\
\hline (iii) Bulk density & Core sampler method (Richards, 1954) \\
\hline (iv) Particle density & Pycnometer method (Black, 1965) \\
\hline (v) Hydraulic conductivity & $\begin{array}{l}\text { Schoonover's method, method No. 34 (b) U.S.D.A. Hbk. } \\
\text { No. } 60\end{array}$ \\
\hline
\end{tabular}


Table.3 Effect of various tree cropping systems on texture (sand, silt, clay \%) of soil

\begin{tabular}{|c|c|c|c|c|c|c|c|c|c|c|}
\hline \multirow{2}{*}{ S. No. } & \multirow{2}{*}{\multicolumn{2}{|c|}{ Treatment / Tree cropping systems }} & \multirow{2}{*}{\multicolumn{4}{|c|}{ 0-15 cm (depth) }} & \multirow{2}{*}{\multicolumn{4}{|c|}{$15-30 \mathrm{~cm}$ (depth) }} \\
\hline & & & & & & & & & & \\
\hline 1 & Annla + Rer & Rasin & $\begin{array}{c}\begin{array}{c}\text { Sand } \\
(\%)\end{array} \\
28.39\end{array}$ & $\begin{array}{c}\text { Silt } \\
(\%)\end{array}$ & $\begin{array}{c}\text { Clay }(\%) \\
20.53\end{array}$ & $\begin{array}{l}\text { Texture } \\
\text { Silt loam }\end{array}$ & $\begin{array}{c}\text { Sand (\%) } \\
25.56\end{array}$ & $\begin{array}{c}\begin{array}{c}\text { Silt } \\
(\%)\end{array} \\
53.50\end{array}$ & \begin{tabular}{|c|} 
Clay $(\%)$ \\
20.94
\end{tabular} & $\begin{array}{l}\text { Texture } \\
\text { Silt loam }\end{array}$ \\
\hline & & Centre & 28.02 & 51.47 & 20.51 & Silt loam & 25.51 & 53.60 & 20.89 & Silt loam \\
\hline \multirow[t]{2}{*}{2} & \multirow[t]{2}{*}{ Aonla + Guava } & Basin & 28.35 & 51.83 & 19.82 & Silt loam & 25.31 & 54.58 & 20.11 & Silt loam \\
\hline & & Centre & 28.20 & 52.01 & 19.79 & Silt loam & 25.07 & 54.85 & 20.08 & Silt loam \\
\hline \multirow[t]{2}{*}{3} & \multirow{2}{*}{ Aonla + Karonda } & Basin & 27.60 & 52.79 & 19.65 & Silt loam & 24.97 & 54.74 & 20.29 & Silt loam \\
\hline & & Centre & 27.07 & 53.31 & 19.62 & Silt loam & 24.05 & 55.70 & 20.25 & Silt loam \\
\hline \multirow[t]{2}{*}{4} & \multirow[t]{2}{*}{ Ber + Guava + Phalsa } & Basin & 28.60 & 50.99 & 20.61 & Silt loam & 26.60 & 52.47 & 20.93 & Silt loam \\
\hline & & Centre & 28.27 & 51.16 & 20.57 & Silt loam & 26.13 & 52.93 & 20.94 & Silt loam \\
\hline \multirow[t]{2}{*}{5} & \multirow[t]{2}{*}{ Aonla + Subabool } & Basin & 28.25 & 51.94 & 19.81 & Silt loam & 25.06 & 54.62 & 20.32 & Silt loam \\
\hline & & Centre & 28.01 & 52.21 & 19.78 & Silt loam & 24.92 & 54.78 & 20.30 & Silt loam \\
\hline \multirow[t]{2}{*}{6} & \multicolumn{2}{|l|}{ Control } & 24.75 & 54.17 & 21.08 & Silt loam & 21.27 & 56.28 & 22.45 & Silt loam \\
\hline & \multicolumn{2}{|l|}{$\mathrm{CD}$ at $5 \%$} & 1.29 & NS & 0.94 & & 1.16 & NS & 0.97 & \\
\hline
\end{tabular}

\begin{tabular}{|c|c|c|c|c|c|c|c|c|c|c|}
\hline \multirow[t]{2}{*}{ S. No. } & \multirow{2}{*}{\multicolumn{2}{|c|}{ Treatment / Tree cropping systems }} & \multirow{2}{*}{\multicolumn{4}{|c|}{$30-45 \mathrm{~cm}$ (depth) }} & \multirow{2}{*}{\multicolumn{4}{|c|}{$45-60 \mathrm{~cm}$ (depth) }} \\
\hline & & & & & & & & & & \\
\hline & & & \multirow{2}{*}{$\begin{array}{c}\begin{array}{c}\text { Sand } \\
(\%)\end{array} \\
21.93\end{array}$} & \multirow{2}{*}{$\begin{array}{c}\begin{array}{c}\text { Silt } \\
(\%)\end{array} \\
56.50\end{array}$} & \multirow{2}{*}{$\begin{array}{c}\text { Clay }(\%) \\
21.57\end{array}$} & \multirow{2}{*}{$\begin{array}{l}\text { Texture } \\
\text { Silt loam }\end{array}$} & \multirow{2}{*}{$\begin{array}{c}\text { Sand (\%) } \\
17.00\end{array}$} & \multirow{2}{*}{$\begin{array}{l}\text { Silt } \\
(\%)\end{array}$} & \multirow{2}{*}{$\begin{array}{c}\text { Clay }(\%) \\
23.75 \\
\end{array}$} & \multirow{2}{*}{$\begin{array}{c}\text { Texture } \\
\text { Silt loam }\end{array}$} \\
\hline 1 & AOIIIC & Dasm & & & & & & & & \\
\hline & & Centre & 21.75 & 56.84 & 21.41 & Silt loam & 19.64 & 57.17 & 23.19 & Silt loam \\
\hline \multirow[t]{2}{*}{2} & \multirow{2}{*}{ Aonla + Guava } & Basin & 21.78 & 56.80 & 21.42 & Silt loam & 19.70 & 58.25 & 22.05 & Silt loam \\
\hline & & Centre & 21.60 & 57.01 & 21.39 & Silt loam & 19.57 & 58.34 & 22.09 & Silt loam \\
\hline \multirow[t]{2}{*}{3} & \multirow[t]{2}{*}{ Aonla + Karonda } & Basin & 20.17 & 57.10 & 22.73 & Silt loam & 18.16 & 58.90 & 22.94 & Silt loam \\
\hline & & Centre & 20.10 & 57.23 & 22.67 & Silt loam & 18.10 & 59.03 & 22.87 & Silt loam \\
\hline \multirow[t]{2}{*}{4} & \multirow[t]{2}{*}{ Ber + Guava + Phalsa } & Basin & 21.84 & 56.43 & 21.93 & Silt loam & 19.86 & 57.50 & 22.64 & Silt loam \\
\hline & & Centre & 21.68 & 56.47 & 21.75 & Silt loam & 19.72 & 57.62 & 22.86 & Silt loam \\
\hline \multirow[t]{2}{*}{5} & \multirow[t]{2}{*}{ Aonla + Subabool } & Basin & 20.61 & 57.00 & 22.39 & Silt loam & 19.60 & 58.70 & 21.70 & Silt loam \\
\hline & & Centre & 20.48 & 57.12 & 22.40 & Silt loam & 19.48 & 58.92 & 21.60 & Silt loam \\
\hline \multirow[t]{2}{*}{6} & \multicolumn{2}{|l|}{ Control } & 18.47 & 58.10 & 22.93 & Silt loam & 17.00 & 59.25 & 23.75 & Silt loam \\
\hline & \multicolumn{2}{|l|}{$\mathrm{CD}$ at $5 \%$} & 0.98 & NS & 1.03 & & 0.89 & NS & 1.06 & \\
\hline
\end{tabular}


Table.4 Effect of various tree cropping systems on water holding capacity (\%) of soil

\begin{tabular}{|c|c|c|c|c|c|c|c|c|}
\hline \multirow[t]{2}{*}{ S. No. } & \multirow{2}{*}{\multicolumn{2}{|c|}{ Treatment / Tree cropping systems }} & \multicolumn{4}{|c|}{ Water holding capacity at different depth $(\mathrm{cm})$} & \multirow{3}{*}{$\begin{array}{c}\text { Mean value }(\mathbf{0 - 6 0} \\
\mathbf{c m})\end{array}$} & \multirow{4}{*}{$\begin{array}{l}\text { Average value of } \\
\text { basin and centre } \\
21.44\end{array}$} \\
\hline & & & 0-15 & 15-30 & 30-45 & 45-60 & & \\
\hline \multirow[t]{2}{*}{1} & \multirow[t]{2}{*}{ Aonla + Ber } & Basin & 23.30 & 22.38 & 21.35 & 20.47 & & \\
\hline & & Centre & 21.90 & 21.20 & 20.62 & 20.30 & 21.01 & \\
\hline \multirow[t]{2}{*}{2} & \multirow[t]{2}{*}{ Aonla + Guava } & Basin & 22.87 & 21.52 & 20.88 & 20.36 & 21.41 & \multirow[t]{2}{*}{21.00} \\
\hline & & Centre & 21.13 & 20.80 & 20.50 & 19.97 & 20.60 & \\
\hline \multirow[t]{2}{*}{3} & \multirow[t]{2}{*}{ Aonla + Karonda } & Basin & 22.07 & 21.34 & 20.68 & 20.15 & 21.06 & \multirow[t]{2}{*}{20.77} \\
\hline & & Centre & 21.04 & 20.65 & 20.33 & 19.91 & 20.48 & \\
\hline \multirow[t]{2}{*}{4} & \multirow{2}{*}{ Ber + Guava + Phalsa } & Basin & 24.06 & 23.10 & 21.60 & 20.89 & 22.39 & \multirow[t]{2}{*}{21.18} \\
\hline & & Centre & 22.18 & 21.40 & 20.76 & 20.53 & 21.22 & \\
\hline \multirow[t]{2}{*}{5} & \multirow[t]{2}{*}{ Aonla + Subabool } & Basin & 22.40 & 21.34 & 20.84 & 19.98 & 21.14 & \multirow[t]{2}{*}{20.83} \\
\hline & & Centre & 21.15 & 20.72 & 20.28 & 19.85 & 20.53 & \\
\hline \multirow[t]{2}{*}{6} & \multicolumn{2}{|l|}{ Control } & 18.57 & 17.98 & 17.46 & 17.15 & 17.79 & 17.79 \\
\hline & \multicolumn{2}{|l|}{$\mathrm{CD}$ at $5 \%$} & 0.68 & 0.66 & 0.63 & 0.62 & & \\
\hline
\end{tabular}

Table.5 Effect of various tree cropping systems on bulk density $(\mathrm{gm} / \mathrm{cc})$ of soil

\begin{tabular}{|c|c|c|c|c|c|c|c|c|}
\hline \multirow[t]{2}{*}{ S. No. } & \multirow{2}{*}{\multicolumn{2}{|c|}{ Treatment / Tree cropping systems }} & \multicolumn{4}{|c|}{ Bulk density at different depth (cm) } & \multirow{3}{*}{$\begin{array}{c}\begin{array}{c}\text { Mean value }(\mathbf{0}-60 \\
\text { cm) }\end{array} \\
1.48\end{array}$} & \multirow{4}{*}{$\begin{array}{l}\text { Average value of } \\
\text { basin and centre } \\
1.51\end{array}$} \\
\hline & & & \multirow{2}{*}{$\begin{array}{r}\mathbf{0 - 1 5} \\
1.40\end{array}$} & \multirow{2}{*}{$\begin{array}{c}\mathbf{1 5 - 3 0} \\
1.47\end{array}$} & \multirow{2}{*}{$\begin{array}{c}30-45 \\
1.50\end{array}$} & \multirow{2}{*}{$\begin{array}{c}\mathbf{4 5 - 6 0} \\
1.54\end{array}$} & & \\
\hline 1 & Aonla + Ber & Basin & & & & & & \\
\hline & & Centre & 1.48 & 1.53 & 1.54 & 1.57 & 1.53 & \\
\hline \multirow[t]{2}{*}{2} & \multirow[t]{2}{*}{ Aonla + Guava } & Basin & 1.46 & 1.50 & 1.52 & 1.56 & 1.51 & \multirow[t]{2}{*}{1.53} \\
\hline & & Centre & 1.50 & 1.53 & 1.56 & 1.58 & 1.54 & \\
\hline \multirow[t]{2}{*}{3} & \multirow[t]{2}{*}{ Aonla + Karonda } & Basin & 1.51 & 1.54 & 1.57 & 1.59 & 1.55 & \multirow[t]{2}{*}{1.56} \\
\hline & & Centre & 1.54 & 1.57 & 1.58 & 1.60 & 1.57 & \\
\hline \multirow[t]{2}{*}{4} & \multirow{2}{*}{ Ber + Guava + Phalsa } & Basin & 1.39 & 1.44 & 1.49 & 1.52 & 1.46 & \multirow[t]{2}{*}{1.49} \\
\hline & & Centre & 1.46 & 1.51 & 1.53 & 1.55 & 1.51 & \\
\hline \multirow[t]{2}{*}{5} & \multirow[t]{2}{*}{ Aonla + Subabool } & Basin & 1.49 & 1.52 & 1.53 & 1.56 & 1.53 & \multirow[t]{2}{*}{1.54} \\
\hline & & Centre & 1.53 & 1.54 & 1.55 & 1.58 & 1.55 & \\
\hline \multirow[t]{2}{*}{6} & \multicolumn{2}{|l|}{ Control } & 1.57 & 1.60 & 1.62 & 1.63 & 1.61 & 1.61 \\
\hline & \multicolumn{2}{|l|}{$\mathrm{CD}$ at $5 \%$} & 0.023 & 0.024 & 0.024 & 0.025 & & \\
\hline
\end{tabular}


Table.4 Effect of various tree cropping systems on particle density (gm/cc) of soil

\begin{tabular}{|c|c|c|c|c|c|c|c|c|}
\hline \multirow[t]{2}{*}{ S. No. } & \multirow{2}{*}{\multicolumn{2}{|c|}{ Treatment / Tree cropping systems }} & \multicolumn{4}{|c|}{ Particle density at different depth (cm) } & \multirow{3}{*}{$\begin{array}{c}\text { Mean value }(\mathbf{0 - 6 0} \\
\mathbf{c m})\end{array}$} & \multirow{4}{*}{$\begin{array}{l}\text { Average value of } \\
\text { basin and centre } \\
2.42\end{array}$} \\
\hline & & & \multirow{2}{*}{$\begin{array}{l}\mathbf{0 - 1 5} \\
2.37\end{array}$} & \multirow{2}{*}{$\begin{array}{c}\mathbf{1 5 - 3 0} \\
2.39\end{array}$} & \multirow{2}{*}{$\begin{array}{c}\mathbf{3 0 - 4 5} \\
2.41\end{array}$} & \multirow{2}{*}{$\begin{array}{c}\mathbf{4 5 - 6 0} \\
2.44\end{array}$} & & \\
\hline 1 & Aonla + Ber & Basin & & & & & & \\
\hline & & Centre & 2.40 & 2.42 & 2.45 & 2.46 & 2.43 & \\
\hline \multirow[t]{2}{*}{2} & \multirow[t]{2}{*}{ Aonla + Guava } & Basin & 2.39 & 2.40 & 2.42 & 2.43 & 2.41 & \multirow[t]{2}{*}{2.42} \\
\hline & & Centre & 2.41 & 2.43 & 2.44 & 2.44 & 2.43 & \\
\hline \multirow[t]{2}{*}{3} & \multirow[t]{2}{*}{ Aonla + Karonda } & Basin & 2.43 & 2.45 & 2.46 & 2.47 & 2.45 & \multirow[t]{2}{*}{2.46} \\
\hline & & Centre & 2.46 & 2.46 & 2.47 & 2.48 & 2.47 & \\
\hline \multirow[t]{2}{*}{4} & \multirow{2}{*}{ Ber + Guava + Phalsa } & Basin & 2.37 & 2.39 & 2.41 & 2.41 & 2.40 & \multirow[t]{2}{*}{2.41} \\
\hline & & Centre & 2.40 & 2.41 & 2.42 & 2.43 & 2.42 & \\
\hline \multirow[t]{2}{*}{5} & \multirow[t]{2}{*}{ Aonla + Subabool } & Basin & 2.39 & 2.41 & 2.41 & 2.43 & 2.41 & \multirow[t]{2}{*}{2.43} \\
\hline & & Centre & 2.42 & 2.44 & 2.45 & 2.47 & 2.45 & \\
\hline \multirow[t]{2}{*}{6} & \multicolumn{2}{|l|}{ Control } & 2.48 & 2.50 & 2.51 & 2.52 & 2.50 & 2.50 \\
\hline & \multicolumn{2}{|l|}{$\mathrm{CD}$ at $5 \%$} & 0.039 & 0.04 & 0.04 & 0.04 & & \\
\hline
\end{tabular}

Table.6 Effect of various tree cropping systems on hydraulic conductivity ( $\mathrm{cm} / \mathrm{hr}$ ) of soil

\begin{tabular}{|c|c|c|c|c|c|c|c|c|}
\hline \multirow[t]{2}{*}{ S. No. } & \multirow{2}{*}{\multicolumn{2}{|c|}{ Treatment / Tree cropping systems }} & \multicolumn{4}{|c|}{ Hydraulic Conductivity at different depth (cm) } & \multirow{3}{*}{$\begin{array}{c}\text { Mean value }(\mathbf{0 - 6 0} \\
\text { cm) }\end{array}$} & \multirow{4}{*}{$\begin{array}{l}\text { Average value of } \\
\text { basin and centre } \\
0.22\end{array}$} \\
\hline & & & $0-15$ & 15-30 & $30-45$ & $45-60$ & & \\
\hline \multirow[t]{2}{*}{1} & \multirow[t]{2}{*}{ Aonla + Ber } & Basin & 0.38 & 0.29 & 0.20 & 0.13 & & \\
\hline & & Centre & 0.27 & 0.23 & 0.15 & 0.10 & 0.19 & \\
\hline \multirow[t]{2}{*}{2} & \multirow[t]{2}{*}{ Aonla + Guava } & Basin & 0.35 & 0.25 & 0.17 & 0.11 & 0.22 & \multirow[t]{2}{*}{0.20} \\
\hline & & Centre & 0.24 & 0.20 & 0.13 & 0.09 & 0.17 & \\
\hline \multirow[t]{2}{*}{3} & \multirow[t]{2}{*}{ Aonla + Karonda } & Basin & 0.28 & 0.21 & 0.14 & 0.09 & 0.18 & \multirow[t]{2}{*}{0.16} \\
\hline & & Centre & 0.19 & 0.15 & 0.11 & 0.07 & 0.13 & \\
\hline \multirow[t]{2}{*}{4} & \multirow{2}{*}{ Ber + Guava + Phalsa } & Basin & 0.41 & 0.34 & 0.21 & 0.14 & 0.28 & \multirow[t]{2}{*}{0.25} \\
\hline & & Centre & 0.32 & 0.25 & 0.16 & 0.12 & 0.21 & \\
\hline \multirow[t]{2}{*}{5} & \multirow[t]{2}{*}{ Aonla + Subabool } & Basin & 0.31 & 0.24 & 0.17 & 0.10 & 0.21 & \multirow[t]{2}{*}{0.19} \\
\hline & & Centre & 0.23 & 0.19 & 0.14 & 0.08 & 0.16 & \\
\hline \multirow[t]{2}{*}{6} & \multicolumn{2}{|l|}{ Control } & 0.05 & 0.039 & 0.032 & 0.02 & 0.04 & 0.04 \\
\hline & \multicolumn{2}{|l|}{$\mathrm{CD}$ at $5 \%$} & 0.005 & 0.004 & 0.003 & 0.002 & & \\
\hline
\end{tabular}




\section{Particle density}

It was found that particle density of soil declined appreciably with all the tree cropping systems at all the depths over control (barren site). It was lowest in Ber + Guava + Phalsa cropping system. Reduction in particle density could improve the physical condition of soil mainly because of increase in organic matter and there by chemical condition of soil also.

\section{Hydraulic conductivity}

Present findings revealed that hydraulic conductivity of soil increased considerably in area planted under different cropping models at all the depths over control (barren site). Basins of plants of different combinations showed higher hydraulic conductivity as compared to centres. With increasing depths of soils, hydraulic conductivity was reduced under different systems. Besides reduction in ESP (Exchangeable Sodium Percentage), increase in porosity is mainly responsible for improvement in hydraulic conductivity of soil as advocated by Dixit and Lal (1972) and Garg and Jain (1992). Yadav and Agrawal (1961) reported that green manuring with Dhaincha was found to hasten the process of soil reclamation by increasing hydraulic conductivity and leaching of sodium salts. Our results are also in conformity with the findings of Singh (1990) and Malviya and Singh (1998) who observed that improvement in hydraulic conductivity of soil is associated with tree plantation. Singh and Jaiswal (1973) reported that the nature and degree of permeability depends on soil texture, nature of the exchangeable cations and composition of the saturating solution.

Based on the results obtained from the present investigation, it is concluded that all the cropping systems improved the soil as compared to control (barren site). Decrease in bulk density, particle density, silt and increase in water holding capacity, hydraulic conductivity, sand percent indicated considerable improvement in physical properties of soil.

Among various tree cropping systems, Ber +Guava + Phalsa emerged most superior in improving the physical characteristics soil and thereby availability of nutrients. Aonla + Ber tree cropping system was found the next best combination in this respect.

Application of research- The findings are very useful to understand the influence of tree plantation in reclaiming the large extent of salt affected soil and converting them into productive one. The tree cropping system, Ber +Guava + Phalsa can be highly useful for reclaiming such type of problematic soil.

\section{References}

Agrawal, R.R. and Gupta, R.N. (1968). Saline Alkali soils in India. Tech-Bull. 15 (Agricultural Series) Indian Council of Agricultural Research, New Delhi.

Black, C.A. (1965). Methods of soil analysis. American Soc. Agron. Madison. Wilconsin, U.S.A.

Chopra, S.L. and Kanwar, J.S. (1976). Analytical Agricultural Chemistry.

Dixit, K.V. and Lal, R.N. (1972). Effect of exchangeable sodium on hydralic conductivity of soils. J. Indian Soc. Soil Sci. 20: 1-5.

Garg, V.K. and Jain, R.K. (1992). Influence of fuel wood trees on sodic soils. Canadian Journal of Forest Research, 22 (5): 729-735.

Kamara, C.S. and Haque, I. (1992). Faidherbia albida and its effects on Ethiopia Highland vertisols. Agroforestry systems, 18: 17-29.

Khan, S.A. (1997-98). Annual Report. AICRP on Agro-forestry. Directrate of 
Research C.S.A.U.A. and T., Kanpur.

Khan, (1966). Effect of cropping system on certain physical properties of soil under irrigated conditions. Pakistan J. Soil Sci. 2 (2): 23-30.

Malviya, S.K. and Singh, I.S. (1998). Effect of fruit based cropping models on chemical properties of soil. Indian $J$. Hort. 55 (3): 236-242.

Richards, L.A. (ed.) (1954). Diagnosis and improvement of saline alkali soils. U.S.D.A. Hand Book No. 60, United States Department of Agriculture, Washington.

Singh, E.N., Nungchim, A., Singh, S.S. and
Tewari, S.C(2001). Influence of Tectona grandis and Duabanga grandiflora on soil properties in humid tropics of Arunachal Pradesh. Indian Journal of Forestry, 24 (2): 135-142.

Singh, I.S. (1990). Mahua-an oil bearing tree. Bull. N.D.U.A.T., Faizabad. pp. 11.

Yadav, J.S.P. and Agrawal, R.R. (1961). A comparative study of effectiveness of gypsum and Dhaincha in the reclamation of saline alkali soils. $J$. Indian Soc. Soil Sci. 9: 151-156.

\section{How to cite this article:}

Pandey, C.S. 2019. Influence of Tree Cropping Systems on Physical Properties of Salt Affected Soil. Int.J.Curr.Microbiol.App.Sci. 8(04): 739-747.

doi: https://doi.org/10.20546/ijcmas.2019.804.080 